\title{
Method for Obtaining Coefficients of Powers of Bivariate Generating Functions
}

\author{
Dmitry Kruchinin $^{1}$ (D), Vladimir Kruchinin ${ }^{2}(\mathbb{D})$ and Yuriy Shablya ${ }^{1, *(D)}$ \\ 1 Department of Complex Information Security of Computer Systems, Tomsk State University of Control \\ Systems and Radioelectronics, 634050 Tomsk, Russia; kdv@fb.tusur.ru \\ 2 Institute of Innovation, Tomsk State University of Control Systems and Radioelectronics, \\ 634050 Tomsk, Russia; kru@2i.tusur.ru \\ * Correspondence: shablya-yv@mail.ru
}

Citation: Kruchinin, D.; Kruchinin, V.; Shablya, Y. Method for Obtaining Coefficients of Powers of Bivariate Generating Functions. Mathematics 2021, 9, 428. https://doi.org/ $10.3390 /$ math 9040428

Academic Editors: Francesco Aldo Costabile, Maria I. Gualtieri and Anna Napoli

Received: 31 December 2020

Accepted: 14 February 2021

Published: 22 February 2021

Publisher's Note: MDPI stays neutral with regard to jurisdictional claims in published maps and institutional affiliations.

Copyright: (C) 2021 by the authors. Licensee MDPI, Basel, Switzerland. This article is an open access article distributed under the terms and conditions of the Creative Commons Attribution (CC BY) license (https:/ / creativecommons.org/licenses/by/ $4.0 /)$.

\begin{abstract}
In this paper, we study methods for obtaining explicit formulas for the coefficients of generating functions. To solve this problem, we consider the methods that are based on using the powers of generating functions. We propose to generalize the concept of compositae to the case of generating functions in two variables and define basic operations on such compositae: composition, addition, multiplication, reciprocation and compositional inversion. These operations allow obtaining explicit formulas for compositae and coefficients of bivariate generating functions. In addition, we present several examples of applying the obtained results for getting explicit formulas for the coefficients of bivariate generating functions. The introduced mathematical apparatus can be used for solving different problems that are related to the theory of generating functions.
\end{abstract}

Keywords: formal power series; composition of generation functions; bivariate generating function; composita; explicit formula

MSC: 05A15; 40B05

\section{Introduction}

Generating functions are a widely used and powerful tool for solving problems in combinatorics, mathematical analysis, statistics, etc. For example, methods of the theory of generating functions are used for solving problems in combinatorics, since generating functions allow obtaining a compact representation of discrete structures and process them. For the first time, methods of generating functions were applied by de Moivre to solve recurrence equations. Next, Euler expanded the methods of generating functions for solving research problems related to partitions. In this case, a generating function through its coefficients shows the value for some special numbers that have combinatorial interpretations. Great contributions to the development of methods of generating functions and their application for solving mathematical problems in combinatorics were made by Riordan [1], Comtet [2], Flajolet and Sedgewick [3], Wilf [4], Stanley [5], Egorychev [6], Lando [7] and other scientists.

Moreover, generating functions are the main means of describing polynomials. Polynomials are one of the basic mathematical objects, and they are used in different areas of pure and applied mathematics. In this case, the coefficients of the generating function for a polynomial show the form of the polynomial for the fixed values of its parameters. Many studies present various properties of polynomials and their generating functions (e.g., those by Boas and Buck [8], Roman [9], Srivastava [10-12] and Simsek [13-15]).

The development of new methods for obtaining explicit formulas for the coefficients of generating functions is relevant research trend. The solution of this problem allows finding explicit formulas for special numbers and polynomials that are described by these generating functions. Different approaches for solving this problem can be found in 
papers by the following authors: Srivastava [16,17], Boyadzhiev [18], Cenkci [19] and other scientists.

Note that many research papers that are devoted to combinatorial problems and generating functions use the coefficients of the powers of generating functions. The coefficients of the powers of generating functions were first obtained by Euler when he obtained the coefficient function for $\left(1+x+x^{2}+\ldots+x^{n}+\ldots\right)^{k}$. The Binomial theorem, which is also the power of the generating function $(x+y)$, was known even before de Moivre and Euler's works were published. In addition, the coefficients of the powers of generating functions play an important role in performing the composition of the generating functions.

The following concepts are related to the coefficients of the powers of generating functions:

1. Potential polynomials introduced by Comtet [2]: The potential polynomial $P_{n}^{(k)}$ is the $k$ th power of an exponential generating function ( $k$ is a complex number):

$$
\left(1+\sum_{n>0} g_{n} \frac{x^{n}}{n !}\right)^{k}=1+\sum_{n>0} P_{n}^{(k)} \frac{x^{n}}{n !} .
$$

For the coefficients of potential polynomials, there is a relationship with the Bell polynomials, but operations on such polynomials are not defined.

2. Riordan arrays introduced by Shapiro et al. [20]: A Riordan array is a pair of generating functions $D=(F(x), G(x))$ where $F(x)=\sum_{n \geq 0} f(n) x^{n}$ and $G(x)=\sum_{n>0} g(n) x^{n}$.

It forms an infinite matrix $D=\left(d_{n, k}\right)_{n, k \geq 0}$ where $d_{n, k}=\left[x^{n}\right] F(x) G(x)^{k}$. If we consider the associated subgroup of the Riordan group $D=(1, G(x))$, then we get $d_{n, k}=\left[x^{n}\right] G(x)^{k}$ or

$$
G(x)^{k}=\sum_{n \geq k} d_{n, k} x^{n}
$$

However, there are no universal rules for obtaining explicit formulas for $d_{n, k}$.

3. Power matrices introduced by Knuth [21]: The power matrix of a given power series $V(x)=V_{1} x+V_{2} x^{2}+\ldots$ is the infinite array of coefficients $v_{n, k}=\frac{n !}{k !}\left[x^{n}\right] V(x)^{k}$. Thus, the $k$ th power of $V(x)$ can be presented in the form

$$
V(x)^{k}=\sum_{n \geq k} \frac{k !}{n !} v_{n, k} x^{n} .
$$

In addition, there are formulas for obtaining the coefficients $w_{n, k}$ for the composition of power series $W(z)=U(V(z))$ and for a power series of the form $W(x)=\alpha U(\beta x)$. However, the development of this mathematical apparatus is not presented.

4. Compositae introduced by Kruchinin [22,23]: The composita $F^{\Delta}(n, k)$ of a generating function $F(x)=\sum_{n>0} f(n, k) x^{n}$ is a coefficients function of its $k$ th power:

$$
F(x)^{k}=\sum_{n \geq k} F^{\Delta}(n, k) x^{n} .
$$

For two generating functions $F(x)$ and $G(x)$ and their compositae $F^{\Delta}(n, k)$ and $G^{\Delta}(n, k)$, we can find the composita $A^{\Delta}(n, k)$ of the generating function $A(x)$ for the following cases:

- $\quad$ addition of generating functions: $A(x)=F(x)+G(x)$;

- multiplication of generating functions: $A(x)=F(x) \cdot G(x)$;

- composition of generating functions: $A(x)=G(F(x))$;

- reciprocation of generating functions: $A(x) \cdot F(x)=1$;

- compositional inversion of generating functions: $F(A(x))=x$.

In this way, it is possible to obtain an explicit formula for the composita for various types of generating functions. 
It is also worth noting the results derived by M. Drmota [24] and aimed at obtaining an asymptotic expansion of the coefficients of the powers of generating functions.

However, all these concepts mainly consider generating functions in one variable, while there are many problems associated with generating functions in several variables (multivariate generating functions). Attempts to systematize the process of finding the coefficients of multivariate generating functions were made by Pemantle et al. [25], but they also investigated asymptotic methods for solving this problem. More on asymptotics derived from multivariate generating functions can be found in [26].

To solve this problem explicitly, we propose to generalize the concept of compositae to the case of generating functions in two variables (bivariate generating functions). The effectiveness of applying compositae is shown in obtaining explicit formulas for the coefficients of many ordinary generating functions that are related to special numbers and polynomials [27-34]. This research aims to improve and extend the original method.

The organization of this paper is as follows. Section 2 is devoted to a brief description of the proposed generalization of the concept of compositae. In Section 3, we introduce basic operations on compositae of bivariate generating functions: composition, addition, multiplication, reciprocation and compositional inversion. To confirm the effectiveness of using the proposed generalization of the concept of compositae, we present several examples of finding explicit formulas for coefficients of bivariate generating functions. The obtained results are shown in Section 4.

\section{Composita of a Multivariate Generating Function}

A multivariate generating function is the following formal power series:

$$
F(x, y, \ldots, z)=\sum_{n \geq 0} \sum_{m \geq 0} \ldots \sum_{l \geq 0} f(n, m, \ldots, k) x^{n} y^{m} \cdots z^{l} .
$$

By $\operatorname{ord}(F)$, we denote the order of a formal power series $F(x, y, \ldots, z)$, which is defined as follows [35]:

$$
\operatorname{ord}(F)= \begin{cases}\min \{r=n+m+\ldots+l: f(n, m, \ldots, l) \neq 0\}, & \text { if } F(x, y, \ldots, z) \neq 0 \\ +\infty, & \text { if } F(x, y, \ldots, z)=0\end{cases}
$$

For two formal power series $F(x, y, \ldots, z)$ and $G(x, y, \ldots, z)$ with $\operatorname{ord}(F)>0$ and $\operatorname{ord}(G)>0$, the order of $F(x, y, \ldots, z) \cdot G(x, y, \ldots, z)$ is

$$
\operatorname{ord}(F \cdot G)=\operatorname{ord}(F)+\operatorname{ord}(G) .
$$

For a formal power series $F(x, y, \ldots, z)$ with $\operatorname{ord}(F)>0$, the order of $F(x, y, \ldots, z)^{k}$, $k \in \mathbb{N}$, satisfies the inequality

$$
\operatorname{ord}\left(F^{k}\right) \geq k .
$$

Next, we consider the coefficients of the $k$ th power of a multivariate generating function

$$
F(x, y, \ldots, z)^{k}=\sum_{n \geq 0} \sum_{m \geq 0} \ldots \sum_{l \geq 0} f(n, m, \ldots, l, k) x^{n} y^{m} \cdots z^{l},
$$

where $k \in \mathbb{N}_{0}$.

In general, to calculate the coefficients $f(n, m, \ldots, l, k)$, we can use the method based on the following formula:

$$
f(n, m, \ldots, l, k)=\sum_{\eta_{1}+\eta_{2}+\ldots+\eta_{k}=n}\left(\sum_{\mu_{1}+\mu_{2}+\ldots+\mu_{k}=m}\left(\ldots\left(\sum_{\lambda_{1}+\lambda_{2}+\ldots+\lambda_{k}=l}\left(\prod_{i=1}^{k} f\left(\eta_{i}, \mu_{i}, \ldots, \lambda_{i}\right)\right)\right) \ldots\right)\right),
$$

where $\eta_{i}, \mu_{i}, \ldots, \lambda_{i} \in \mathbb{N}_{0}$. 
Definition 1. The composita $F^{\Delta}(n, m, \ldots, l, k)$ of a multivariate generating function

$$
F(x, y, \ldots, z)=\sum_{n \geq 0} \sum_{m \geq 0} \ldots \sum_{l \geq 0} f(n, m, \ldots, k) x^{n} y^{m} \cdots z^{l}, \quad \operatorname{ord}(F) \geq 1,
$$

is a coefficients function of the $k$ th power of the generating function $F(x, y, \ldots, z)$ :

$$
F(x, y, \ldots, z)^{k}=\sum_{n \geq 0} \sum_{m \geq 0} \ldots \sum_{l \geq 0} F^{\Delta}(n, m, \ldots, l, k) x^{n} y^{m} \cdots z^{l} .
$$

In addition, we set the following condition: $F(x, y, \ldots, z)^{0}=1$.

Hence, the composita $F^{\Delta}(n, m, \ldots, l, k)$ for $k=0$ is defined as follows:

$$
F^{\Delta}(n, m, \ldots, l, 0)= \begin{cases}1, & \text { if } n=m=\ldots=l=0 \\ 0, & \text { otherwise }\end{cases}
$$

We can also write a recurrence for calculating compositae for $k>0$

$$
F^{\Delta}(n, m, \ldots, l, k)= \begin{cases}f(n, m, \ldots, l), & \text { if } k=1 \\ \sum_{i=0}^{n} \sum_{j=0}^{m} \ldots \sum_{s=0}^{l} f(i, j, \ldots, s) F^{\Delta}(n-i, m-j, \ldots, l-s, k-1), & \text { if } k>1\end{cases}
$$

that is based on using the convolution operation for

$$
F(x, y, \ldots, z)^{k}=F(x, y, \ldots, z) F(x, y, \ldots, z)^{k-1}, \quad F(x, y, \ldots, z)^{0}=1 .
$$

\section{Operations on Compositae of Bivariate Generating Functions}

A bivariate generating function is the following formal power series:

$$
F(x, y)=\sum_{n \geq 0} \sum_{m \geq 0} f(n, m) x^{n} y^{m}
$$

Using the concept of compositae, the $k$ th power of the bivariate generating function $F(x, y)$, with $\operatorname{ord}(F) \geq 1$, can be presented as follows:

$$
F(x, y)^{k}=\sum_{n \geq 0} \sum_{m \geq 0} F^{\Delta}(n, m, k) x^{n} y^{m} .
$$

Next, we introduce basic operations on compositae of bivariate generating functions: composition, addition, multiplication, reciprocation and compositional inversion. These operations allow obtaining explicit formulas for compositae and coefficients of bivariate generating functions.

\subsection{Composition of Bivariate Generating Functions}

Theorem 1. Suppose that:

$$
\begin{gathered}
H(x, y)=\sum_{n \geq 0} \sum_{m \geq 0} h(n, m) x^{n} y^{m}, \\
A(x, y)^{k}=\sum_{n \geq 0} \sum_{m \geq 0} A^{\Delta}(n, m, k) x^{n} y^{m}, \quad \operatorname{ord}(A) \geq 1, \\
B(x, y)^{k}=\sum_{n \geq 0} \sum_{m \geq 0} B^{\Delta}(n, m, k) x^{n} y^{m}, \quad \operatorname{ord}(B) \geq 1 .
\end{gathered}
$$


Then, the coefficients $g(n, m)$ of the composition of the bivariate generating functions

$$
G(x, y)=H(A(x, y), B(x, y))=\sum_{n \geq 0} \sum_{m \geq 0} g(n, m) x^{n} y^{m}
$$

are equal to

$$
g(n, m)=\sum_{k_{a}=0}^{n+m} \sum_{k_{b}=0}^{n+m-k_{a}} h\left(k_{a}, k_{b}\right) \sum_{i=0}^{n} \sum_{j=0}^{m} A^{\Delta}\left(i, j, k_{a}\right) B^{\Delta}\left(n-i, m-j, k_{b}\right) .
$$

Proof. Consider the given composition of bivariate generating functions

$$
G(x, y)=H(A(x, y), B(x, y))=\sum_{n \geq 0} \sum_{m \geq 0} h(n, m) A(x, y)^{n} B(x, y)^{m} .
$$

Next, we represent the end part of this generating function as follows:

$$
C(x, y)=A(x, y)^{k_{a}} B(x, y)^{k_{b}}=\sum_{n \geq 0} \sum_{m \geq 0} c\left(n, m, k_{a}, k_{b}\right) x^{n} y^{m} .
$$

To obtain an explicit formula for the coefficients $c\left(n, m, k_{a}, k_{b}\right)$, we apply the convolution operation and get

$$
c\left(n, m, k_{a}, k_{b}\right)=\sum_{i=0}^{n} \sum_{j=0}^{m} A^{\Delta}\left(i, j, k_{a}\right) B^{\Delta}\left(n-i, m-j, k_{b}\right) .
$$

Using Equation (1) and Inequality (2), we also have

$$
\operatorname{ord}\left(A(x, y)^{n} B(x, y)^{m}\right) \geq n+m .
$$

Then, taking into account the restriction for the indices of summation

$$
k_{a}+k_{b} \leq n+m,
$$

we can construct the following two summation schemes for obtaining the coefficients $g(n, m)$ :

$$
g(n, m)=\sum_{k_{a}=0}^{n+m} \sum_{k_{b}=0}^{n+m-k_{a}} h\left(k_{a}, k_{b}\right) c\left(n, m, k_{a}, k_{b}\right)
$$

or

$$
g(n, m)=\sum_{k_{b}=0}^{n+m} \sum_{k_{a}=0}^{n+m-k_{b}} h\left(k_{a}, k_{b}\right) c\left(n, m, k_{a}, k_{b}\right) .
$$

Combining the formula for $g(n, m)$ with Equation (4), we obtain the desired result presented in Equation (3).

Table 1 presents the obtained results for special cases of using the results of Theorem 1 for a bivariate generating function $G(x, y)$ that is presented as the composition of bivariate and ordinary generating functions.

Next, we consider the process of finding coefficients for the $k$ th power of a bivariate generating function that is obtained using the composition of bivariate generating functions. This result can be derived by generalizing the bivariate generating function $H(x, y)$ from Theorem 1 to the case of its $k$ th power. The obtained result is presented in Theorem 2. 
Table 1. Special cases of using the results of Theorem 1.

\begin{tabular}{|c|c|}
\hline Composition & Coefficient \\
\hline$G(x, y)=H(A(x, y), B(x, y))$ & $g(n, m)=\sum_{k_{a}=0}^{n+m} \sum_{k_{b}=0}^{n+m-k_{a}} h\left(k_{a}, k_{b}\right) \sum_{i=0}^{n} \sum_{j=0}^{m} A^{\Delta}\left(i, j, k_{a}\right) B^{\Delta}\left(n-i, m-j, k_{b}\right)$ \\
\hline$G(x, y)=H(A(x, y), B(y))$ & $g(n, m)=\sum_{k_{a}=0}^{n+m} \sum_{k_{b}=0}^{n+m-k_{a}} h\left(k_{a}, k_{b}\right) \sum_{j=0}^{m} A^{\Delta}\left(n, j, k_{a}\right) B^{\Delta}\left(m-j, k_{b}\right)$ \\
\hline$G(x, y)=H(A(y), B(x, y))$ & $g(n, m)=\sum_{k_{a}=0}^{n+m} \sum_{k_{b}=0}^{n+m-k_{a}} h\left(k_{a}, k_{b}\right) \sum_{j=0}^{m} A^{\Delta}\left(j, k_{a}\right) B^{\Delta}\left(n, m-j, k_{b}\right)$ \\
\hline$G(x, y)=H(A(x, y), B(x))$ & $g(n, m)=\sum_{k_{a}=0}^{n+m} \sum_{k_{b}=0}^{n+m-k_{a}} h\left(k_{a}, k_{b}\right) \sum_{i=0}^{n} A^{\Delta}\left(i, m, k_{a}\right) B^{\Delta}\left(n-i, k_{b}\right)$ \\
\hline$G(x, y)=H(A(x), B(x, y))$ & $g(n, m)=\sum_{k_{a}=0}^{n+m} \sum_{k_{b}=0}^{n+m-k_{a}} h\left(k_{a}, k_{b}\right) \sum_{i=0}^{n} A^{\Delta}\left(i, k_{a}\right) B^{\Delta}\left(n-i, m, k_{b}\right)$ \\
\hline$G(x, y)=H(A(x, y), y)$ & $g(n, m)=\sum_{k_{a}=0}^{n+m} \sum_{k_{b}=0}^{n+m-k_{a}} h\left(k_{a}, k_{b}\right) A^{\Delta}\left(n, m-k_{b}, k_{a}\right)$ \\
\hline$G(x, y)=H(y, B(x, y))$ & $g(n, m)=\sum_{k_{a}=0}^{n+m} \sum_{k_{b}=0}^{n+m-k_{a}} h\left(k_{a}, k_{b}\right) B^{\Delta}\left(n, m-k_{a}, k_{b}\right)$ \\
\hline$G(x, y)=H(A(x, y), x)$ & $g(n, m)=\sum_{k_{a}=0}^{n+m} \sum_{k_{b}=0}^{n+m-k_{a}} h\left(k_{a}, k_{b}\right) A^{\Delta}\left(n-k_{b}, m, k_{a}\right)$ \\
\hline$G(x, y)=H(x, B(x, y))$ & $g(n, m)=\sum_{k_{a}=0}^{n+m} \sum_{k_{b}=0}^{n+m-k_{a}} h\left(k_{a}, k_{b}\right) B^{\Delta}\left(n-k_{a}, m, k_{b}\right)$ \\
\hline$G(x, y)=H(A(x), B(y))$ & $g(n, m)=\sum_{k_{a}=0}^{n} \sum_{k_{b}=0}^{m} h\left(k_{a}, k_{b}\right) A^{\Delta}\left(n, k_{a}\right) B^{\Delta}\left(m, k_{b}\right)$ \\
\hline$G(x, y)=H(A(x), y)$ & $g(n, m)=\sum_{k=0}^{n} h(k, m) A^{\Delta}(n, k)$ \\
\hline$G(x, y)=H(x, B(y))$ & $g(n, m)=\sum_{k=0}^{m} h(n, k) B^{\Delta}(m, k)$ \\
\hline$G(x, y)=H(A(x, y))$ & $g(n, m)=\sum_{k=0}^{n+m} h(k) A^{\Delta}(n, m, k)$ \\
\hline
\end{tabular}

Theorem 2. Suppose that:

$$
\begin{gathered}
H(x, y)^{k}=\sum_{n \geq 0} \sum_{m \geq 0} h(n, m, k) x^{n} y^{m}, \\
A(x, y)^{k}=\sum_{n \geq 0} \sum_{m \geq 0} A^{\Delta}(n, m, k) x^{n} y^{m}, \quad \operatorname{ord}(A) \geq 1, \\
B(x, y)^{k}=\sum_{n \geq 0} \sum_{m \geq 0} B^{\Delta}(n, m, k) x^{n} y^{m}, \quad \operatorname{ord}(B) \geq 1 .
\end{gathered}
$$

Then, the coefficients $g(n, m, k)$ of the $k$ th power of the composition of the bivariate generating functions

$$
G(x, y)^{k}=H(A(x, y), B(x, y))^{k}=\sum_{n \geq 0} \sum_{m \geq 0} g(n, m, k) x^{n} y^{m}
$$

are equal to

$$
g(n, m, k)=\sum_{k_{a}=0}^{n+m} \sum_{k_{b}=0}^{n+m-k_{a}} h\left(k_{a}, k_{b}, k\right) \sum_{i=0}^{n} \sum_{j=0}^{m} A^{\Delta}\left(i, j, k_{a}\right) B^{\Delta}\left(n-i, m-j, k_{b}\right) .
$$

Proof. The proof of Theorem 2 is similar to the proof of Theorem 1.

Corollary 1. Suppose that:

$$
H(x, y)^{k}=\sum_{n \geq 0} \sum_{m \geq 0} H^{\Delta}(n, m, k) x^{n} y^{m}, \quad \operatorname{ord}(H) \geq 1,
$$




$$
\begin{aligned}
& A(x, y)^{k}=\sum_{n \geq 0} \sum_{m \geq 0} A^{\Delta}(n, m, k) x^{n} y^{m}, \quad \operatorname{ord}(A) \geq 1, \\
& B(x, y)^{k}=\sum_{n \geq 0} \sum_{m \geq 0} B^{\Delta}(n, m, k) x^{n} y^{m}, \quad \operatorname{ord}(B) \geq 1 .
\end{aligned}
$$

Then, the composita $G^{\Delta}(n, m, k)$ of the composition of the bivariate generating functions

$$
G(x, y)=H(A(x, y), B(x, y))=\sum_{n \geq 0} \sum_{m \geq 0} g(n, m) x^{n} y^{m}
$$

is equal to

$$
G^{\Delta}(n, m, k)=\sum_{k_{a}=0}^{n+m} \sum_{k_{b}=0}^{n+m-k_{a}} H^{\Delta}\left(k_{a}, k_{b}, k\right) \sum_{i=0}^{n} \sum_{j=0}^{m} A^{\Delta}\left(i, j, k_{a}\right) B^{\Delta}\left(n-i, m-j, k_{b}\right) .
$$

Corollary 1 can be applied for calculating the composita of a given bivariate generating function based on its decomposition into simpler functions. For example, it can be used for addition or multiplication of bivariate generating functions.

\subsection{Addition of Bivariate Generating Functions}

Theorem 3. Suppose that:

$$
\begin{aligned}
& A(x, y)^{k}=\sum_{n \geq 0} \sum_{m \geq 0} A^{\Delta}(n, m, k) x^{n} y^{m}, \quad \operatorname{ord}(A) \geq 1, \\
& B(x, y)^{k}=\sum_{n \geq 0} \sum_{m \geq 0} B^{\Delta}(n, m, k) x^{n} y^{m}, \quad \operatorname{ord}(B) \geq 1 .
\end{aligned}
$$

Then, the composita $G^{\Delta}(n, m, k)$ of the addition of the bivariate generating functions

$$
G(x, y)=A(x, y)+B(x, y)=\sum_{n \geq 0} \sum_{m \geq 0} g(n, m) x^{n} y^{m}
$$

is equal to

$$
G^{\Delta}(n, m, k)=\sum_{k_{a}=0}^{n+m}\left(\begin{array}{c}
k \\
k_{a}
\end{array}\right) \sum_{i=0}^{n} \sum_{j=0}^{m} A^{\Delta}\left(i, j, k_{a}\right) B^{\Delta}\left(n-i, m-j, k-k_{a}\right) .
$$

Proof. Consider a bivariate generating function

$$
H(x, y)=x+y
$$

and its $k$ th power, that is based on the binomial theorem,

$$
H(x, y)^{k}=(x+y)^{k}=\sum_{n \geq 0} \sum_{m \geq 0} H^{\Delta}(n, m, k) x^{n} y^{m}=\sum_{n \geq 0} \sum_{m \geq 0}\left(\begin{array}{l}
k \\
n
\end{array}\right) \delta(m, k-n) x^{n} y^{m},
$$

where $\delta(i, j)$ is the Kronecker delta function:

$$
\delta(i, j)= \begin{cases}0, & \text { if } i \neq j \\ 1, & \text { if } i=j\end{cases}
$$

Applying Equation (6) for the composition of generating functions

$$
G(x, y)=A(x, y)+B(x, y)=H(A(x, y), B(x, y)),
$$


we obtain

$$
G^{\Delta}(n, m, k)=\sum_{k_{a}=0}^{n+m} \sum_{k_{b}=0}^{n+m-k_{a}}\left(\begin{array}{c}
k \\
k_{a}
\end{array}\right) \delta\left(k_{b}, k-k_{a}\right) \sum_{i=0}^{n} \sum_{j=0}^{m} A^{\Delta}\left(i, j, k_{a}\right) B^{\Delta}\left(n-i, m-j, k_{b}\right) .
$$

Using the properties of the Kronecker delta function, we get $k_{b}=k-k_{a}$.

Simplifying the formula for $G^{\Delta}(n, m, k)$, we obtain the desired result presented in Equation (7).

\subsection{Multiplication of Bivariate Generating Functions}

Theorem 4. Suppose that:

$$
\begin{aligned}
& A(x, y)^{k}=\sum_{n \geq 0} \sum_{m \geq 0} A^{\Delta}(n, m, k) x^{n} y^{m}, \quad \operatorname{ord}(A) \geq 1, \\
& B(x, y)^{k}=\sum_{n \geq 0} \sum_{m \geq 0} B^{\Delta}(n, m, k) x^{n} y^{m}, \quad \operatorname{ord}(B) \geq 1 .
\end{aligned}
$$

Then, the composita $G^{\Delta}(n, m, k)$ of the addition of the bivariate generating functions

$$
G(x, y)=A(x, y) \cdot B(x, y)=\sum_{n \geq 0} \sum_{m \geq 0} g(n, m) x^{n} y^{m}
$$

is equal to

$$
G^{\Delta}(n, m, k)=\sum_{i=0}^{n} \sum_{j=0}^{m} A^{\Delta}(i, j, k) B^{\Delta}(n-i, m-j, k) .
$$

Proof. Consider a bivariate generating function

$$
H(x, y)=x y
$$

and its $k$ th power

$$
H(x, y)^{k}=(x y)^{k}=\sum_{n \geq 0} \sum_{m \geq 0} H^{\Delta}(n, m, k) x^{n} y^{m}=\sum_{n \geq 0} \sum_{m \geq 0} \delta(n, k) \delta(m, k) x^{n} y^{m} .
$$

Applying Equation (6) for the composition of generating functions

$$
G(x, y)=A(x, y) \cdot B(x, y)=H(A(x, y), B(x, y)),
$$

we obtain

$$
G^{\Delta}(n, m, k)=\sum_{k_{a}=0}^{n+m} \sum_{k_{b}=0}^{n+m-k_{a}} \delta\left(k_{a}, k\right) \delta\left(k_{b}, k\right) \sum_{i=0}^{n} \sum_{j=0}^{m} A^{\Delta}\left(i, j, k_{a}\right) B^{\Delta}\left(n-i, m-j, k_{b}\right) .
$$

Using the properties of the Kronecker delta function, we get $k_{a}=k, k_{b}=k$.

Simplifying the formula for $G^{\Delta}(n, m, k)$, we obtain the desired result presented in Equation (8).

\subsection{Reciprocation of Bivariate Generating Functions}

A reciprocal generating function $G(x, y)$ of a bivariate generating function

$$
F(x, y)=\sum_{n \geq 0} \sum_{m \geq 0} f(n, m) x^{n} y^{m}
$$

is a formal power series such that satisfies the condition

$$
F(x, y) \cdot G(x, y)=1 .
$$


Theorem 5. Suppose that:

$$
\begin{gathered}
F(x, y)=\sum_{n \geq 0} \sum_{m \geq 0} f(n, m) x^{n} y^{m}, \quad f(0,0) \neq 0, \quad F(x, y)^{k}=\sum_{n \geq 0} \sum_{m \geq 0} f(n, m, k) x^{n} y^{m}, \\
G(x, y)=\sum_{n \geq 0} \sum_{m \geq 0} g(n, m) x^{n} y^{m}, \quad g(0,0) \neq 0, \quad G(x, y)^{k}=\sum_{n \geq 0} \sum_{m \geq 0} g(n, m, k) x^{n} y^{m}, \\
F(x, y) \cdot G(x, y)=1 .
\end{gathered}
$$

Then, the coefficients $g(n, m, k)$ are equal to

$$
g(n, m, k)=\sum_{i=0}^{n+m}\left(\begin{array}{c}
n+m+k \\
i+k
\end{array}\right)\left(\begin{array}{c}
i+k-1 \\
i
\end{array}\right) \frac{(-1)^{i}}{f(0,0)^{i+k}} f(n, m, i) .
$$

Proof. Consider the $k$ th power of the generating function $G(x, y)$ as the following composition of generating functions:

$$
G(x, y)^{k}=\left(\frac{1}{F(x, y)}\right)^{k}=\frac{1}{f(0,0)^{k}}\left(\frac{1}{1+\left(\frac{F(x, y)}{f(0,0)}-1\right)}\right)^{k}=\frac{1}{f(0,0)^{k}} H(A(x, y))^{k},
$$

where

$$
\begin{gathered}
H(x)=\frac{1}{1+x}, \quad H(x)^{k}=\sum_{n \geq 0} h(n, k) x^{n}, \\
A(x, y)=\frac{F(x, y)}{f(0,0)}-1, \quad A(x, y)^{k}=\sum_{n \geq 0} \sum_{m \geq 0} A^{\Delta}(n, m, k) x^{n} y^{m} .
\end{gathered}
$$

The coefficients $h(n, k)$ can be calculating by

$$
h(n, k)=(-1)^{n}\left(\begin{array}{c}
n+k-1 \\
n
\end{array}\right) .
$$

Using the binomial theorem, the composita $A^{\Delta}(n, m, k)$ is equal to

$$
A^{\Delta}(n, m, k)=\sum_{i=0}^{k}\left(\begin{array}{l}
k \\
i
\end{array}\right) \frac{f(n, m, i)}{f(0,0)^{i}}(-1)^{k-i} .
$$

Applying Equation (5) for $G(x, y)$, we obtain

$$
g(n, m, k)=\frac{1}{f(0,0)^{k}} \sum_{k_{a}=0}^{n+m} h\left(k_{a}, k\right) A^{\Delta}\left(n, m, k_{a}\right)=\sum_{k_{a}=0}^{n+m} \sum_{i=0}^{k_{a}}\left(\begin{array}{c}
k_{a}+k-1 \\
k_{a}
\end{array}\right)\left(\begin{array}{c}
k_{a} \\
i
\end{array}\right) \frac{f(n, m, i)}{f(0,0)^{i+k}}(-1)^{i} .
$$

Then, we change the order of summation and get

$$
g(n, m, k)=\sum_{i=0}^{n+m} \sum_{k_{a}=0}^{n+m-i}\left(\begin{array}{c}
k_{a}+i+k-1 \\
k_{a}+i
\end{array}\right)\left(\begin{array}{c}
k_{a}+i \\
i
\end{array}\right) \frac{f(n, m, i)}{f(0,0)^{i+k}}(-1)^{i} .
$$

To remove the coefficient $k_{a}$ from the right binomial coefficient, we transform the binomial coefficient and obtain

$$
g(n, m, k)=\sum_{i=0}^{n+m} \sum_{k_{a}=0}^{n+m-i}\left(\begin{array}{c}
k_{a}+i+k-1 \\
k_{a}
\end{array}\right)\left(\begin{array}{c}
i+k-1 \\
i
\end{array}\right) \frac{f(n, m, i)}{f(0,0)^{i+k}}(-1)^{i} .
$$


Next, we can simplify this formula by using the following identity (Identity (1.49) in [36]):

$$
\sum_{k_{a}=0}^{n+m-i}\left(\begin{array}{c}
k_{a}+i+k-1 \\
k_{a}
\end{array}\right)=\left(\begin{array}{c}
n+m+k \\
n+m-i
\end{array}\right)
$$

Thus, we obtain the desired result presented in Equation (9).

\subsection{Compositional Inversion of Bivariate Generating Functions}

A compositional inverse $\bar{F}(x, y)$ of a bivariate generating function

$$
F(x, y)=\sum_{n \geq 0} \sum_{m \geq 0} f(n, m) x^{n} y^{m}
$$

with respect to the variable $x$ is a formal power series such that satisfies the condition

$$
F(\bar{F}(x, y), y)=x
$$

Theorem 6. Suppose that:

$$
\begin{gathered}
F(x, y)=\sum_{n \geq 0} \sum_{m \geq 0} f(n, m) x^{n} y^{m}, \quad f(0,0)=0, \quad F(x, y)^{k}=\sum_{n \geq 0} \sum_{m \geq 0} F^{\Delta}(n, m, k) x^{n} y^{m}, \\
\bar{F}(x, y)=\sum_{n \geq 0} \sum_{m \geq 0} \bar{f}(n, m) x^{n} y^{m}, \quad \bar{f}(0,0)=0, \quad \bar{F}(x, y)^{k}=\sum_{n \geq 0} \sum_{m \geq 0} \bar{F}^{\Delta}(n, m, k) x^{n} y^{m}, \\
F(\bar{F}(x, y), y)=x .
\end{gathered}
$$

Then, the composita $\bar{F}^{\Delta}(n, m, k)$ is equal to

$$
\bar{F}^{\Delta}(n, m, k)=\frac{k}{n} \sum_{i=0}^{n+m}\left(\begin{array}{c}
2 n+m-k \\
i+n
\end{array}\right)\left(\begin{array}{c}
i+n-1 \\
i
\end{array}\right) \frac{(-1)^{i}}{f(1,0)^{i+n}} F^{\Delta}(i+n-k, m, i) .
$$

Proof. Using the Lagrange inversion theorem [37] for the functional equation

$$
\bar{F}(x, y)=x G(\bar{F}(x, y), y),
$$

where

$$
G(x, y)=\sum_{n \geq 0} \sum_{m \geq 0} g(n, m) x^{n} y^{m}, \quad g(0,0) \neq 0, \quad G(x, y)^{k}=\sum_{n \geq 0} \sum_{m \geq 0} g(n, m, k) x^{n} y^{m},
$$

we obtain

$$
\bar{F}^{\Delta}(n, m, k)=\frac{k}{n} g(n-k, m, n) .
$$

In addition, we can represent this functional equation as follows:

$$
\begin{gathered}
x=\frac{\bar{F}(x, y)}{G(\bar{F}(x, y), y)}=F(\bar{F}(x, y), y), \\
F(x, y)=\frac{x}{G(x, y)} .
\end{gathered}
$$

Applying Equation (9) for $G(x, y)$, we get the coefficients of its $k$ th power

$$
g(n, m, k)=\sum_{i=0}^{n+m}\left(\begin{array}{c}
n+m+k \\
i+k
\end{array}\right)\left(\begin{array}{c}
i+k-1 \\
i
\end{array}\right) \frac{(-1)^{i}}{f(1,0)^{i+k}} F^{\Delta}(i+n, m, i) .
$$

Combining Equation (11) with Equation (12), we obtain the desired result presented in Equation (10). 


\section{Application of Compositae for Obtaining Coefficients of Bivariate Generating Functions}

Next, we present several examples of applying the obtained results for getting explicit formulas for coefficients of bivariate generating functions.

Example 1. First, let consider the following simple composition of generating functions:

$$
G(x, y)=\sum_{n \geq 0} \sum_{m \geq 0} g(n, m) x^{n} y^{m}=H(A(x, y))=H(x+y) .
$$

The composita of the generating function $A(x, y)=x+y$ is

$$
A^{\Delta}(n, m, k)=\left(\begin{array}{l}
k \\
n
\end{array}\right) \delta(m, k-n) .
$$

Applying Theorem 1, we can obtain the coefficients $g(n, m)$ of the generating function $G(x, y)$

$$
g(n, m)=\sum_{k=0}^{n+m} h(k) A^{\Delta}(n, m, k)=\sum_{k=0}^{n+m} h(k)\left(\begin{array}{l}
k \\
n
\end{array}\right) \delta(m, k-n)=h(n+m)\left(\begin{array}{c}
n+m \\
n
\end{array}\right) .
$$

If we have the generating function

$$
H(x)=\sum_{n \geq 0} h(n) x^{n}=\sum_{n \geq 0} x^{n}=\frac{1}{1-x}
$$

then we get

$$
\begin{gathered}
G(x, y)=H(x+y)=\frac{1}{1-x-y^{\prime}} \\
g(n, m)=h(n+m)\left(\begin{array}{c}
n+m \\
n
\end{array}\right)=\left(\begin{array}{c}
n+m \\
n
\end{array}\right) .
\end{gathered}
$$

If we have the generating function

$$
H(x)=\sum_{n \geq 0} h(n) x^{n}=\sum_{n \geq 0} \frac{1}{n !} x^{n}=e^{x},
$$

then we get

$$
\begin{gathered}
G(x, y)=H(x+y)=e^{x+y} \\
g(n, m)=h(n+m)\left(\begin{array}{c}
n+m \\
n
\end{array}\right)=\frac{1}{(n+m) !}\left(\begin{array}{c}
n+m \\
n
\end{array}\right)=\frac{1}{n ! m !} .
\end{gathered}
$$

If we have the generating function

$$
H(x)=\sum_{n>0} h(n) x^{n}=\sum_{n>0} \frac{(-1)^{n-1}}{n} x^{n}=\log (1+x),
$$

then we get

$$
\begin{gathered}
G(x, y)=H(x+y)=\log (1+x+y), \\
g(n, m)=h(n+m)\left(\begin{array}{c}
n+m \\
n
\end{array}\right)=\frac{(-1)^{n+m-1}}{n+m}\left(\begin{array}{c}
n+m \\
n
\end{array}\right), \quad g(0,0)=0 .
\end{gathered}
$$

If we have the generating function of the Catalan numbers (the sequence A000108 in OEIS [38])

$$
H(x)=\sum_{n \geq 0} C_{n} x^{n}=\sum_{n \geq 0} \frac{1}{n+1}\left(\begin{array}{c}
2 n \\
n
\end{array}\right) x^{n}=\frac{1-\sqrt{1-4 x}}{2 x},
$$


then we get

$$
\begin{gathered}
G(x, y)=H(x+y)=\frac{1-\sqrt{1-4(x+y)}}{2(x+y)} \\
g(n, m)=h(n+m)\left(\begin{array}{c}
n+m \\
n
\end{array}\right)=C_{n+m}\left(\begin{array}{c}
n+m \\
n
\end{array}\right)=\frac{1}{n+m+1}\left(\begin{array}{c}
2 n+2 m \\
n+m
\end{array}\right)\left(\begin{array}{c}
n+m \\
n
\end{array}\right) .
\end{gathered}
$$

Example 2. Let consider the generating function of the Eulerian numbers (the sequence A173018 in OEIS [38])

$$
E(x, y)=\sum_{n \geq 0} \sum_{m \geq 0} \frac{E_{n, m}}{n !} x^{n} y^{m}=\frac{y-1}{y-e^{x(y-1)}} .
$$

Consider this generating function $E(x, y)$ as the following composition of generating functions:

$$
E(x, y)=\frac{y-1}{y-e^{x(y-1)}}=\frac{x(y-1)}{x(y-1)-x\left(e^{x(y-1)}-1\right)}=\frac{1}{1-x \frac{e^{x(y-1)}-1}{x(y-1)}}=H(A(x, y)),
$$

where

$$
\begin{gathered}
H(x)=\sum_{n \geq 0} h(n) x^{n}=\sum_{n \geq 0} x^{n}=\frac{1}{1-x}, \\
A(x, y)=\frac{x \frac{e^{x(y-1)}-1}{x(y-1)}=B(x, C(x, y)),}{B(x, y)=\frac{x}{y}\left(e^{y}-1\right),} \\
C(x, y)=x(y-1) .
\end{gathered}
$$

Using the identity for the Stirling numbers of the second kind

$$
\left(e^{x}-1\right)^{k}=\sum_{n \geq k}\left\{\begin{array}{l}
n \\
k
\end{array}\right\} \frac{k !}{n !} x^{n},
$$

we get the composita of the generating function $B(x, y)$

$$
B^{\Delta}(n, m, k)=\left\{\begin{array}{c}
m+k \\
k
\end{array}\right\} \frac{k !}{(m+k) !} \delta(n, k) .
$$

Using the binomial theorem for

$$
(x y-x)^{k}=\sum_{m \geq 0}\left(\begin{array}{c}
k \\
m
\end{array}\right)(x y)^{m}(-x)^{k-m}=\sum_{m \geq 0}\left(\begin{array}{c}
k \\
m
\end{array}\right) x^{k} y^{m}(-1)^{k-m},
$$

we get the composita of the generating function $C(x, y)$

$$
C^{\Delta}(n, m, k)=\left(\begin{array}{c}
k \\
m
\end{array}\right)(-1)^{k-m} \delta(n, k) .
$$

Combining the obtained results and applying Theorem 1 for the composition $B(x, C(x, y))$, we obtain the composita of the generating function $A(x, y)$

$$
\begin{gathered}
A^{\Delta}(n, m, k)=\sum_{k_{a}=0}^{n+m} \sum_{k_{b}=0}^{n+m-k_{a}} B^{\Delta}\left(k_{a}, k_{b}, k\right) C^{\Delta}\left(n-k_{a}, m, k_{b}\right) \\
=\sum_{k_{a}=0}^{n+m} \sum_{k_{b}=0}^{n+m-k_{a}}\left\{\begin{array}{c}
k_{b}+k \\
k
\end{array}\right\} \frac{k !}{\left(k_{b}+k\right) !} \delta\left(k_{a}, k\right)\left(\begin{array}{c}
k_{b} \\
m
\end{array}\right)(-1)^{k_{b}-m} \delta\left(n-k_{a}, k_{b}\right) .
\end{gathered}
$$

Using the properties of the Kronecker delta function, we get $k_{a}=k, k_{b}=n-k$. 
Simplifying the formula for $A^{\Delta}(n, m, k)$, we obtain

$$
A^{\Delta}(n, m, k)=\left\{\begin{array}{l}
n \\
k
\end{array}\right\}\left(\begin{array}{c}
n-k \\
m
\end{array}\right) \frac{k !}{n !}(-1)^{n-k-m} .
$$

Applying Theorem 1 for the composition $H(A(x, y))$, we can obtain the following well-known explicit formula for the Eulerian numbers ([39], Equation (6.40)):

$$
E_{n, m}=n ! \sum_{k=0}^{n+m} h(k) A^{\Delta}(n, m, k)=\sum_{k=0}^{n}\left\{\begin{array}{l}
n \\
k
\end{array}\right\}\left(\begin{array}{c}
n-k \\
m
\end{array}\right)(-1)^{n-k-m} k ! .
$$

Example 3. Let consider the generating function of the Euler-Catalan numbers [40] (the sequence A316773 in OEIS [38])

$$
E C(x, y)=\sum_{n \geq 0} \sum_{m \geq 0} \frac{E C_{n, m}}{n !} x^{n} y^{m}=\frac{y-1}{y-e^{C(x)(y-1)}},
$$

where

$$
C(x)=\frac{1-\sqrt{1-4 x}}{2}
$$

is the generating function of the Catalan numbers. tions:

Consider this generating function $E C(x, y)$ as the following composition of generating func-

$$
E C(x, y)=E(C(x), y),
$$

where $E(x, y)$ is the generating function of the Eulerian numbers.

The composita of the generating function $C(x)$ is (cf. [22])

$$
C^{\Delta}(n, k)=\frac{k}{n}\left(\begin{array}{c}
2 n-k-1 \\
n-1
\end{array}\right) .
$$

Applying Theorem 1 for the composition $\operatorname{EC}(C(x), y)$, we can obtain the following explicit formula for the Euler-Catalan numbers:

$$
E C_{n, m}=n ! \sum_{k=0}^{n} E(k, m) C^{\Delta}(n, k)=n ! \sum_{k=0}^{n} \frac{E_{k, m}}{k !} \frac{k}{n}\left(\begin{array}{c}
2 n-k-1 \\
n-1
\end{array}\right)=\sum_{k=m+1}^{n} E_{k, m} \frac{(2 n-k-1) !}{(k-1) !(n-k) !} .
$$

Example 4. Let consider the generating function of the number triangle that forms the sequence A064189 in OEIS [38]

$$
G(x, y)=\sum_{n \geq 0} \sum_{m \geq 0} g(n, m) x^{n} y^{m}=\frac{2}{1-x-2 x y+\sqrt{1-2 x-3 x^{2}}}=\frac{M(x)}{1-x y M(x)},
$$

where

$$
M(x)=\frac{1-x-\sqrt{1-2 x-3 x^{2}}}{2 x^{2}}
$$

is the generating function of the Motzkin numbers (the sequence A001006 in OEIS [38]).

The elements $g(n, m)$ of this number triangle define the number of lattice paths from $(0,0)$ to $(n, m)$, staying weakly above the $x$-axis and consisting of steps $(1,1),(1,-1)$ and $(1,0)$.

Consider this generating function $G(x, y)$ as the following composition of generating functions:

$$
G(x, y)=\frac{H(x, y)}{x y}=\frac{B\left(M_{x y}(x, y)\right)}{x y},
$$


where

$$
\begin{gathered}
H(x, y)=\sum_{n>0} \sum_{m>0} h(n, m) x^{n} y^{m}=\frac{x y M(x)}{1-x y M(x)}=\frac{M_{x y}(x, y)}{1-M_{x y}(x, y)}=B\left(M_{x y}(x, y)\right), \\
B(x)=\sum_{n>0} b(n) x^{n}=\sum_{n>0} x^{n}=\frac{x}{1-x}, \\
M_{x y}(x, y)=x y M(x) .
\end{gathered}
$$

The generating function $M(x)$ satisfies the following functional equation:

$$
M(x)=1+x M(x)+x^{2} M(x) .
$$

This functional equation can be transformed into

$$
M_{x}(x)=x A\left(M_{x}(x)\right)
$$

where

$$
\begin{gathered}
A(x)=1+x+x^{2}, \\
M_{x}(x)=x M(x) .
\end{gathered}
$$

Using the binomial theorem for

$$
\left(1+x+x^{2}\right)^{k}=\sum_{j=0}^{k}\left(\begin{array}{c}
k \\
j
\end{array}\right)\left(x+x^{2}\right)^{j}=\sum_{j=0}^{k}\left(\begin{array}{l}
k \\
j
\end{array}\right) x^{j} \sum_{n=0}^{j}\left(\begin{array}{l}
j \\
n
\end{array}\right) x^{n}=\sum_{n \geq 0} \sum_{j=0}^{k}\left(\begin{array}{c}
k \\
j
\end{array}\right)\left(\begin{array}{c}
j \\
n-j
\end{array}\right) x^{n},
$$

we get the coefficients of the $k$ th power of the generating function $A(x)$

$$
a(n, k)=\sum_{j=0}^{k}\left(\begin{array}{l}
k \\
j
\end{array}\right)\left(\begin{array}{c}
j \\
n-j
\end{array}\right) .
$$

Using the Lagrange inversion theorem for (13), we obtain the composita of the generating function $M_{x}(x)$

$$
M_{x}^{\Delta}(n, k)=\frac{k}{n} a(n-k, n) .
$$

Hence, the composita of the generating function $M_{x y}(x, y)$ is

$$
M_{x y}^{\Delta}(n, m, k)=M_{x}^{\Delta}(n, k) \delta(m, k) .
$$

Applying Theorem 1 for the composition $B\left(M_{x y}(x, y)\right)$, we obtain the coefficients $h(n, m)$ of the generating function $H(x, y)$

$$
h(n, m)=\sum_{k=0}^{n+m} b(k) M_{x y}^{\Delta}(n, m, k)=\sum_{k=1}^{n+m} M_{x}^{\Delta}(n, k) \delta(m, k)=M_{x}^{\Delta}(n, m) .
$$

Finally, we get the coefficients $g(n, m)$ of the generating function $G(x, y)$

$$
g(n, m)=h(n+1, m+1)=\frac{m+1}{n+1} \sum_{j=0}^{n-m}\left(\begin{array}{c}
n+1 \\
j
\end{array}\right)\left(\begin{array}{c}
j \\
n-m-j
\end{array}\right) .
$$

Example 5. Let consider the generating function of the number triangle that forms the sequence A336524 in OEIS [38]

$$
G(x, y)=\sum_{n \geq 0} \sum_{m \geq 0} g(n, m) x^{n} y^{m}=\frac{1-\sqrt{1-4 x-4 x y}}{2 x} .
$$


The elements $g(n, m)$ of this number triangle define the number of unlabeled binary trees with $n$ internal nodes and exactly $m$ distinguished external nodes.

Consider this generating function $G(x, y)$ as the following composition of generating functions:

$$
G(x, y)=\frac{H(x, y)}{x}=\frac{C(A(x, y))}{x},
$$

where

$$
\begin{gathered}
H(x, y)=\sum_{n>0} \sum_{m \geq 0} h(n, m) x^{n} y^{m}=\frac{1-\sqrt{1-4 x-4 x y}}{2}=C(A(x, y)), \\
C(x)=\sum_{n>0} c(n) x^{n}=\sum_{n>0} C_{n-1} x^{n}=\frac{1-\sqrt{1-4 x}}{2}, \\
A(x, y)=x+x y .
\end{gathered}
$$

Using the binomial theorem for

$$
(x+x y)^{k}=\sum_{m \geq 0}\left(\begin{array}{c}
k \\
m
\end{array}\right) x^{k-m}(x y)^{m}=\sum_{m \geq 0}\left(\begin{array}{c}
k \\
m
\end{array}\right) x^{k} y^{m},
$$

we get the composita of the generating function $A(x, y)$

$$
A^{\Delta}(n, m, k)=\left(\begin{array}{c}
k \\
m
\end{array}\right) \delta(n, k) .
$$

Applying Theorem 1 for the composition $C(A(x, y))$, we obtain the coefficients $h(n, m)$ of the generating function $H(x, y)$

$$
h(n, m)=\sum_{k=0}^{n+m} c(k) A^{\Delta}(n, m, k)=\sum_{k=1}^{n+m} C_{k-1}\left(\begin{array}{c}
k \\
m
\end{array}\right) \delta(n, k)=C_{n-1}\left(\begin{array}{c}
n \\
m
\end{array}\right) .
$$

Finally, we get the coefficients $g(n, m)$ of the generating function $G(x, y)$

$$
g(n, m)=h(n+1, m)=C_{n}\left(\begin{array}{c}
n+1 \\
m
\end{array}\right)=\frac{1}{n+1}\left(\begin{array}{c}
2 n \\
n
\end{array}\right)\left(\begin{array}{c}
n+1 \\
m
\end{array}\right) .
$$

\section{Conclusions}

This paper is devoted to the study of methods for obtaining explicit formulas for the coefficients of generating functions. To solve this problem, we consider the methods that are based on using the powers of generating functions. We propose to generalize the concept of compositae to the case of generating functions in two variables and define basic operations on such compositae: composition, addition, multiplication, reciprocation and compositional inversion. These operations allow obtaining explicit formulas for compositae and coefficients of bivariate generating functions. In addition, we present several examples of applying the obtained results for getting explicit formulas for coefficients of bivariate generating functions.

The introduced mathematical apparatus can be used for solving different problems that are related to the theory of generating functions. For example, it contributes to obtaining new explicit formulas for polynomials and special numbers. In addition, it can be applied for obtaining explicit formulas for the cardinality functions of combinatorial sets. This task is relevant in combinatorics, discrete mathematics and computer science when it is necessary to develop combinatorial generation algorithms [41].

Author Contributions: Investigation, D.K., V.K. and Y.S.; methodology, D.K.; writing-original draft preparation, V.K. and Y.S.; and writing-review and editing, D.K. All authors have read and agreed to the published version of the manuscript. 
Funding: The reported study was funded by RFBR, project number 20-31-70037.

Institutional Review Board Statement: Not applicable.

Informed Consent Statement: Not applicable.

Data Availability Statement: Not applicable.

Acknowledgments: The authors would like to thank the referees for their helpful comments and suggestions.

Conflicts of Interest: The authors declare no conflict of interest.

\section{References}

1. Riordan, J. An Introduction to Combinatorial Analysis; Princeton University Press: Princeton, NJ, USA, 1980.

2. Comtet, L. Advanced Combinatorics; D. Reidel Publishing Company: Dordrecht, The Netherlands, 1974.

3. Flajolet, P.; Sedgewick, R. Analytic Combinatorics; Cambridge University Press: Cambridge, UK, 2009.

4. Wilf, H.S. Generatingfunctionology; Academic Press: Cambridge, MA, USA, 1994.

5. Stanley, R. Enumerative Combinatorics, 2nd ed.; Cambridge University Press: New York, NY, USA, 2012.

6. Egorychev, G.P.; Zima, E.V. Integral representation and algorithms for closed form summation. In Handbook of Algebra; Hazewinkel, M., Ed.; Elsevier: Amsterdam, The Netherlands, 2008; Volume 5, pp. 459-529.

7. Lando, S.K. Lectures on Generating Functions; American Mathematical Society: Providence, RI, USA, 2003.

8. Boas, R.P.J.; Buck, R.C. Polynomial Expansions of Analytic Functions; Springer: Berlin/Heidelberg, Germany, 1958.

9. Roman, S. The Umbral Calculus; Academic Press: Cambridge, MA, USA, 1984.

10. Srivastava, H.M.; Manocha, H.L. A Treatise on Generating Functions (Mathematics and Its Applications); Ellis Horwood: Hemel Hempstead, UK, 1984.

11. Ozden, H.; Simsek, Y.; Srivastava, H.M. A unified presentation of the generating functions of the generalized Bernoulli, Euler and Genocchi polynomials. Comput. Math. Appl. 2010, 60, 2779-2787. [CrossRef]

12. Srivastava, H.M. Some generalizations and basic (or $q$-) extensions of the Bernoulli, Euler and Genocchi polynomials. Appl. Math Inf. Sci. 2011, 5, 390-444.

13. Simsek, Y.; Acikgoz, M. A new generating function of $(q-)$ Bernstein-type polynomials and their interpolation function. Abstr. Appl. Anal. 2010, 2010, 769095. [CrossRef]

14. Simsek, Y. Complete sum of products of $(h, q)$-extension of Euler polynomials and numbers. J. Differ. Equ. Appl. 2010, 16, 1331-1348. [CrossRef]

15. Dere, R.; Simsek, Y. Applications of umbral algebra to some special polynomials. Adv. Stud. Contemp. Math. 2012, 22, 433-438.

16. Srivastava, H.M.; Todorov, P.G. An explicit formula for the generalized Bernoulli polynomials. J. Math. Anal. Appl. 1988, 130, 509-513. [CrossRef]

17. Liu, G.D.; Srivastava, H.M. Explicit formulas for the Norlund polynomials $B_{n}^{(x)}$ and $b_{n}^{(x)}$. Comput. Math. Appl. 2006, 51, 1377-1384. [CrossRef]

18. Boyadzhiev, K.N. Derivative polynomials for tanh, tan, sech and sec in explicit form. Fibonacci Quart. 2007, 45, $291-303$.

19. Cenkci, M. An explicit formula for generalized potential polynomials and its applications. Discret. Math. 2009, 309, 1498-1510. [CrossRef]

20. Shapiro, L.W.; Getu, S.; Woan, W.J.; Woodson, L.C. The Riordan group. Discret. Appl. Math. 1991, 34, 229-239. [CrossRef]

21. Knuth, D.E. The Art of Computer Programming, Volume 2: Seminumerical Algorithms, 3rd ed.; Addison-Wesley Professional: Boston, MA, USA, 1997.

22. Kruchinin, D.V.; Kruchinin, V.V. A method for obtaining generating functions for central coefficients of triangles. J. Integer Seq. 2012, 15, 12.9.3.

23. Kruchinin, D.V.; Kruchinin, V.V. Application of a composition of generating functions for obtaining explicit formulas of polynomials. J. Math. Anal. Appl. 2013, 404, 161-171. [CrossRef]

24. Drmota, M. A bivariate asymptotic expansion of coefficients of powers of generating functions. Eur. J. Combin. 1994, 15, 139-152. [CrossRef]

25. Asymptotics of Multivariate Sequences. Available online: www.cs.auckland.ac.nz/ mcw/Research/mvGF/asymultseq/ (accessed on 1 February 2021).

26. Pemantle, R.; Wilson, M.C. Twenty combinatorial examples of asymptotics derived from multivariate generating functions. SIAM Rev. 2008, 50, 199-272. [CrossRef]

27. Kruchinin, D.V.; Kruchinin, V.V. A method for obtaining expressions for polynomials based on a composition of generating functions. In Proceedings of the International Conference of Numerical Analysis and Applied Mathematics (ICNAAM 2012), Kos, Greece, 19-25 September 2012; Volume 1479, pp. 383-386. [CrossRef]

28. Kruchinin, D.V.; Kruchinin, V.V. Explicit formulas for some generalized polynomials. Appl. Math. Inf. Sci. 2013, 7, 2083-2088 [CrossRef]

29. Kruchinin, D.V.; Shablya, Y.V. Explicit formulas for Meixner polynomials. Int. J. Math. Math. Sci. 2015, 2015, 620569. [CrossRef] 
30. Dewi, I.P.; Utama, S.; Aminah, S. Deriving the explicit formula of Chebyshev polynomials of the third kind and the fourth kind. AIP Conf. Proc. 2017, 2023, 020202. [CrossRef]

31. Kruchinin, D.V. Explicit formulas for Korobov polynomials. Proc. Jangjeon Math. Soc. 2017, 20, 43-50. [CrossRef]

32. Cambazard, H. Fixed-parameter algorithms for rectilinear Steiner tree and rectilinear traveling salesman problem in the plane. Eur. J. Oper. Res. 2018, 270, 419-429. [CrossRef]

33. Banderier, C.; Krattenthaler, C.; Krinik, A.; Kruchinin, D.; Kruchinin, V.; Nguyen, D.; Wallner, M. Explicit formulas for enumeration of lattice paths: Basketball and the kernel method. In Lattice Path Combinatorics and Applications; Springer: Berlin/Heidelberg, Germany, 2019; pp. 78-118.

34. Simsek, Y. Peters type polynomials and numbers and their generating functions: Approach with $p$-adic integral method. Math Methods Appl. Sci. 2019, 42, 7030-7046. [CrossRef]

35. Gan, X.X.; Bugajewski, D. A note on formal power series. Comment. Math. Univ. Carolin. 2010, 51, 595-604.

36. Gould, H.W. Combinatorial Identities: A standardized Set of Tables Listing 500 Binomial Coefficient Summations, 3rd ed.; Morgantown Printing: Morgantown, WV, USA, 1972.

37. Gessel, I.M. A combinatorial proof of the multivariable Lagrange inversion formula. Combin. Theory Ser. A 1987, 45, 178-195. [CrossRef]

38. Sloane, N.J.A. The On-Line Encyclopedia of Integer Sequences. Available online: www.oeis.org (accessed on 1 February 2021).

39. Graham, R.L.; Knuth, D.E.; Patashnik, O. Concrete Mathematics, 2nd ed.; Addison-Wesley: Boston, MA, USA, 1994.

40. Shablya, Y.; Kruchinin, D. Euler-Catalan's number triangle and its application. Symmetry 2020, 12, 600. [CrossRef]

41. Shablya, Y.; Kruchinin, D.; Kruchinin, V. Method for developing combinatorial generation algorithms based on AND/OR trees and its application. Mathematics 2020, 8, 962. [CrossRef] 